\title{
MANUAL THERAPY AS AN ALTERNATIVE TREATMENT FOR PANIC ATTACKS
}

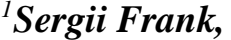 \\ ${ }^{2}$ Michael Frank, \\ ${ }^{3}$ George Frank
}

'Ukraine, Kyiv, Medical Center "Ukrainian Trauma Center"

ORCID ID: https://orcid.org/0000-0002-0053-6898.

Orthopedic traumatologist, manual therapist;

${ }^{2}$ Ukraine, Kyiv, Medical Center "Ukrainian Trauma Center"

ORCID ID: https://orcid.org/0000-0001-5145-9290.

Intern, manual therapist;

${ }^{3}$ Ukraine, Kyiv, Medical Center "Ukrainian Trauma Center"

ORCID ID: https://orcid.org/0000-0003-2377-1337.

Paramedic, manual therapist; $6^{\text {th }}$ year student at the Bogomolets National Medical University

\section{DOI: https://doi.org/10.31435/rssglobal_ws/31082019/6633}

\section{ARTICLE INFO}

Received: 25 June 2019

Accepted: 11 August 2019

Published: 31 August 2019

\section{KEYWORDS}

panic attack, manual therapy, shock wave therapy.

\begin{abstract}
In worldwide medical practice panic attacks are treated mainly by psychological methods and medication. Cognitive-behavioral therapy combined with selective serotonin reuptake inhibitors is considered to be the most effective method of their treatment. However, it has been proven that about a third of patients with panic attacks are treatment resistant. Such people continue experiencing panic attacks and various other symptoms of panic disorder even after receiving the aforementioned treatment. It is this group of patients who are being targeted by the proposed research - manual therapy as an alternative treatment for panic attacks.
\end{abstract}

Citation: Sergii Frank, Michael Frank, George Frank. (2019) Manual Therapy as an Alternative Treatment for Panic Attacks. World Science. 8(48), Vol.2. doi: 10.31435/rsglobal_ws/31082019/6633

Copyright: (C) 2019 Sergii Frank, Michael Frank, George Frank. This is an open-access article distributed under the terms of the Creative Commons Attribution License (CC BY). The use, distribution or reproduction in other forums is permitted, provided the original author(s) or licensor are credited and that the original publication in this journal is cited, in accordance with accepted academic practice. No use, distribution or reproduction is permitted which does not comply with these terms.

Introduction. Panic attacks are sudden periods of intense fear which can include heart palpitations, sweating, trembling, shortness of breath, numbness or a feeling of impending doom [1,2]. Panic attacks can occur due to a number of disorders, including panic disorder, social anxiety disorder, post-traumatic stress disorder, drug use disorder, depression and medical problems [1, 22]. Panic attacks are distinguished from other forms of anxiety by their intensity and their sudden, episodic nature [5]. ICD11 (version dated 04/2019) describes a panic attack (PA) (MB23.H) as: "A discrete episode of intense fear or apprehension accompanied by the rapid and concurrent onset of a number of characteristic symptoms. These symptoms may include, but are not limited to, palpitations or increased heart rate, sweating, trembling, sensations of shortness of breath, feelings of choking, chest pain, nausea or abdominal distress, feelings of dizziness or lightheadedness, chills or hot flushes, tingling or lack of sensation in extremities (i.e., paresthesias), depersonalization or derealization, fear of losing control or going mad, and fear of imminent death. Panic attacks can appear out of the blue or can be triggered by particular situations" [14].

The DSM-5 diagnostic criteria for panic attacks include a discrete period of intense fear or discomfort during which four (or more) of the following symptoms suddenly develop and reach a peak within a few minutes: palpitations, pounding heart, or accelerated heart rate; sweating; trembling or shaking; sensations of shortness of breath or smothering; feelings of choking; chest pain or discomfort; nausea or abdominal distress; feeling dizzy, unsteady, light-headed, or faint; derealization (feeling of 
unreality) or depersonalization (being detached from one-self); fear of losing control or "going crazy"; fear of dying; paresthesias (numbness or tingling sensations); chills or heat sensations [1].

Symptoms of panic attacks reach their maximum within a few minutes [1], the attack itself lasts for an average of 30 minutes, although its duration can vary from seconds to hours [4]. Panic attacks themselves are not life threatening [11, 12]. At the same time, PA patients are at high risk of suicide [1].

Treatment of panic attacks should be directed at the underlying cause [11]. World medical practice suggests psychological therapies and medications for the patients with frequent attacks [5, 19]. Breathing training and muscle relaxation techniques are also being used [20].

Cognitive-behavioral therapy (TF-CBT) is considered to be most effective [7], followed by selective serotonin reuptake inhibitors (SSRI) [3] and psychoanalytic psychotherapy [18]. Often, the desired effect can be achieved by lifestyle changes [22] as well as special breathing exercises [6, 8, 13, 17].

Drug treatments are usually used throughout the duration of panic attack symptoms and discontinued after the patient has been free of symptoms for at least six months [16, 22].

In Europe, about 3\% of the population suffer from panic attacks, in the US it is about 11\% [1]. Panic attacks are more common in women than men [1]. They often begin in puberty or early adulthood [1].

Roughly one-third of PA patients are treatment-resistant [10]. These people continue to have panic attacks and various other panic disorder symptoms after receiving treatment [10]. It is this group of patients who are being targeted by the proposed research - manual therapy as an alternative treatment for panic attacks.

The purpose of this research is to study the effects of manual therapy on panic attacks that are resistant to treatment by conventional methods.

\section{Materials and methods.}

A retrospective analysis of treatment results was conducted for 7 patients with panic attacks who underwent manual therapy treatment at MC "Ukrainian Trauma Center" during the period from January 2016 to June 2019. The age of patients ranged from 15 to 68 years old (their average age being 41.7 years old). At the time of treatment at MC "Ukrainian Trauma Center", these patients had already been diagnosed with panic attacks at other medical institutions, often with concomitant somatic and neurological pathologies. All of them had various lengths of unsuccessful experience in treating their panic attacks at various medical institutions.

The control group consisted of 10 patients from among the core patients of MC "Ukrainian Trauma Center" with vertebrogenic spinal disorders including panic attacks. However, these patients had never been specially treated for panic attacks prior to the current research.

The assessment of patients at MC "Ukrainian Trauma Center" involved their examination by an orthopedic traumatologist and a manual therapist, a spinal MRI and some other tests as needed. The treatment was carried out according to the original method authored by S. Frank. It is based on the best achievements of K. Levit's European school of manual therapy, further supplemented by the author's own developments and achievements of his Ukrainian colleagues. K. Levit's European school of manual therapy sees the prerequisites for manual treatment in the dysfunction of the motor system. Manipulations are considered to be the most adequate method of treatment for reversible functional movement restrictions [15 p. 20]. The goal of manipulative treatment is to restore the function of the joint where it has been inhibited (blocked) [15, p. 23]. The objects of manipulation are functional disorders, mainly blockages in the joints. Blockage refers to a restriction of movement in the motor segment [15, p. 20].

The original method of treatment introduced by S. Frank is protected by scientific publications in European medical journals [21]. The essence of this method is the combination of manual therapy with shock wave therapy (SWT). Combining these two components in a single method creates a synergistic effect [21]. Upon collecting the patient's history, this method involves the following stages: 1) using palpation and an SWT handpiece to precisely locate blocked spinal segments and trigger points; 2) using an SWT handpiece to reduce pain sensitivity in the target area, improve trophic function of the nerve, increase blood circulation in the area of the blocked spinal segment; 3) application of manual therapy techniques to eliminate pathologies with periodic repetition of treatment procedures until a positive clinical result can be obtained.

This method has been successfully practiced over the past ten years including its application for the treatment of panic attacks. 
Results and their discussion. The main group of the study constituted seven patients with panic attacks resistant to conventional methods of treatment. The control group consisted of 10 patients with vertebrogenic spinal disorders and a history of panic attacks with no prior treatment.

The results of the study showed that all patients with panic attacks, both from the main and control group, had vertebrogenic spinal disorders in different parts of the spine. In the main group of the study, one patient was affected by thoracolumbar idiopathic scoliosis and another one - by thoracolumbar pain disorder $(28.6 \%)$; one patient suffered from scoliotic posture and lateral cervical scoliosis (14.3\%); one patient had thoracalgia $(14,3 \%)$ and another one - cervical thoracic pain disorder $(14,3 \%)$; finally cervicothoracolumbar pain disorder was identified in the last two patients $(28,6 \%)$. In the control group, the distribution of vertebral pathologies was as follows: thoracolumbar pain disorder 3 patients $(30 \%)$, cervicalgia -2 patients $(20 \%)$, thoracalgia -2 patients $(20 \%)$, cervical thoracic pain disorder -1 patient $(10 \%)$, cervicothoracolumbar pain disorder -2 patients $(20 \%)$.

All patients of the main and control groups underwent treatment consisting of 3-10 sessions of manual correction of target areas supplemented by shock wave therapy when necessary. After receiving treatment, the patients were observed at MC "Ukrainian Trauma Center" for 1-3 years. As a result of the first course of treatment, panic attacks ceased and never reappeared in five out of seven patients (71\%) of the main group, one patient (14.3\%) had two relapses: in two months and then in 3.5 years after the initial treatment. One patient (14.3\%) continues to suffer from panic attacks, however the interval between the attacks lengthened from 5-10 days to 1-2 months and their intensity decreased by $50 \%$. Recurring panic attacks can now be stopped with 1-2 sessions of manual therapy.

In the control group, panic attacks ceased and never reappeared during 1-3 years of observation in eight $(80 \%)$ out of ten patients after the first course of treatment, one patient $(10 \%)$ had a single relapse during the observation period of 2 years which was successfully treated with one session of manual therapy. One patient (10\%) with a severe vertebrogenic cervicothoracolumbar pain disorder also suffering from panic attacks keeps addressing MC "Ukrainian Trauma Center" every 2-3 months with complaints of fear of dying, accelerated heart rate, dizziness and nausea. The said patient undergoes 2-3 sessions of manual adjustment and receives temporary relief.

Thus, both in the main group with patients suffering from panic attacks resistant to conventional methods of treatment and in the control group where patients have never been treated for panic attacks before, highly meaningful clinical results were observed with respectively $71 \%$ and $80 \%$ success rates in complete elimination of panic attacks throughout the period of observation. In patients with relapses, it was still possible to significantly reduce both the frequency and intensity of panic attacks. It should be noted that the proposed method is effective in cases where conventional treatment for panic attacks turned out to be of no help. The truth of this assertion is proved by the fact that the main group sample consisted of patients suffering from treatment resistant panic attacks.

As outlined above, it proved possible, through manual techniques and without medication or psychological therapies, to achieve meaningful results exceeding the effectiveness of generally accepted conventional treatment.

\section{Clinical case No. 1}

Female patient A aged 26 y.o., lecturer of English at a university. First presented to MC "Ukrainian Trauma Center" in May 2016. Diagnosis: panic attacks.

Complaints: feeling of heaviness in the back, inability to take a deep breath, suddenly and simultaneously occurring: palpitations (often at night time while asleep), trembling, feeling of choking, chills, loss of sensation in the hands and feet, insurmountable fear of going crazy or dying (presence of 6 diagnostic criteria for panic attacks specified in DSM-5 and ICD-11).

Medical history: first became ill in October 2015, after suffering a viral infection started having pain in the thoracic spine accompanied by palpitations and shortness of breath. First panic attack suddenly occurred on October 14-th and lasted for 30 minutes. Subsequent panic attacks occurred at a rate of 2-3 times per week. After medical examination and once a number of possible therapeutic and neurological diagnoses has been eliminated, the patient was referred to a psychiatrist, diagnosed with panic attacks and prescribed medication treatment (benzodiazepines and antidepressants) as well as psychotherapy sessions. As a result of the treatment, the frequency of panic attacks decreased to 2-3 times per month, their intensity somewhat weakened, but new symptoms such as drowsiness, lethargy and memory problems appeared instead. Ongoing panic attacks led the patient to seek treatment at MC "Ukrainian Trauma Center". 
Clinical data: 15 points on the Glasgow Coma Scale, hypesthesia in hands and feet; a spinal MRI revealed degenerative \& dystrophic changes complicated in the thoracic spine by left-sided paramedian protrusions at Th7-Th8, Th8-Th9 and Th10-Th11 levels. X-ray examination confirmed thoracolumbar idiopathic scoliosis.

Treatment: on May 16-th, 18-th, 20-th, 27-th followed by June 10-th and 24-th, 2016, several sessions of manual therapy were carried out which involved adjustments to all parts of the spine. Special attention has been paid to removing spinal blockages in the middle thoracic spine on level Th4-Th5.

After the above treatment, the patient's heart rate decreased to 68 beats per minute, trembling, feeling of choking and chills all disappeared, sensitivity in the hands and feet was restored and her fear ceased. The last low intensity panic attack in this patient was observed on June 9-th, 2016. It lasted only for seven minutes and occurred due to domestic stress. The patient underwent follow-up examinations on June 19-th, 2017 and April 22-nd, 2019. No more panic attacks were observed during this period.

\section{Clinical case No. 2}

Male patient B aged 15 y.o., high school student, athlete, member of the Ukrainian youth rugby team. First presented to MC "Ukrainian Trauma Center" on June 6-th, 2018. Diagnosis: multiple consequences of a sports injury to the spine and back, vegetative-vascular dystonia (vascular dysautonomia) with panic attacks, juvenile hypertension.

Complaints: thoracic back pain, sudden palpitations, chest pain, attacks of groundless fear, claustrophobia, sudden vasospasms with skin blanching and abrupt elevations of blood pressure up to 180/110, trembling, feeling of choking, nausea and abdominal distress, dizziness, fainting, paresthesias (presence of 8 diagnostic criteria for panic attacks specified in DSM-5 and ICD-11).

Medical history: became ill in January 2018 due to a sports injury to the back sustained during athletic competitions. After the initial back injury that occurred on January 11-th, the symptoms gradually increased, the first panic attack occurred at home on January 22-nd, 2018. The patient was taken to the Children's Clinical Hospital No. 6, underwent medical examination and a course of medication and physiotherapy treatment. The patient was discharged on January 29-th with a slight improvement and a diagnosis of vegetative-vascular dystonia (vascular dysautonomia) accompanied by panic attacks and juvenile hypertension. Panic attacks with abrupt elevations of blood pressure continued almost daily thereafter, during the period from January to June the patient was three times admitted to different hospitals due to unceasing panic attacks.

Clinical data: 15 points on the Glasgow Coma Scale, spinal blockages on C4 \& C5 levels, trembling, fear of medical manipulations. A spinal MRI revealed degenerative and dystrophic changes complicated by dorsal protrusions at Th5-Th6, Th6-Th7 \& Th11-Th12 levels in the thoracic spine. A panoramic X-ray of the spine revealed scoliotic posture, lateral scoliosis at C4-C5 and synecorative reflex, therefore showing a classic picture of sports-related PTSD.

Treatment: on June 6-th, 8-th and 15-th, 2016, several manual therapy sessions were carried out which involved adjustments to all parts of the spine and knee joints, special attention was paid to removing vertebral blockages at C4/C5 level. In the course of treatment, the frequency of panic attacks decreased and their intensity waned. No more panic attacks occurred after June 13-th, the blood pressure settled at 110/70 and the heart rate - at 60 beats per minute, chest pain, fear, trembling, feeling of choking, nausea, abdominal distress, dizziness, fainting, paresthesias all disappeared. Follow-up examinations of the patient conducted on December 14-th, 2018 and June 10-th, 2019 confirmed the absence of relapses, the patient returned to training and active sports.

\section{Clinical case No. 3}

Female patient I., 45 y.o., entrepreneur. First presented to MC "Ukrainian Trauma Center" in November 2018. Diagnosis: mitral valve prolapse, myocarditis, premature ventricular contractions, hypotonic-type vegetative-vascular dystonia (vascular dysautonomia), panic attacks, vertebrogenic cervical thoracic pain disorder, chronic cholecystic pancreatitis.

Complaints: pain and constant discomfort in the left side of the chest, extrasystoles, weakness, inability to take a deep breath, sudden trembling, feeling of choking, chills, attacks of groundless fear (presence of 6 diagnostic criteria for panic attacks specified in DSM-5 and ICD-11).

Medical history: became ill in October 2004, after suffering a tracheobronchitis, started experiencing pain on the left side of the chest in the heart area, extrasystoles, shortness of breath, weakness, sweating, fluctuations of blood pressure from 85/60 up to 140/110, back pain, attacks of fear. The patient was hospitalized multiple times for treatment of her heart-related conditions, but no 
significant improvement occurred. She presented to MC "Ukrainian Trauma Center" seeking treatment for her spinal disorders.

Clinical data: a spinal MRI revealed multiple degenerative and dystrophic changes complicated by: C5-C6 \& C7-Th1 disk protrusions in the cervical spine; spondylosis in C4-C5, C5-C6 \& C6-C7 segments; left-sided paramedian protrusions of the Th7-Th8 \& Th10-Th11 discs in the thoracic region of the spine; marginal bone osteophytes along the locking plates of vertebral bodies and facet joints in the range of Th7-Th12; hemangiomas of the vertebral body L4 and the right arch of the vertebral body L3 in the lumbosacral spine.

Treatment: on November 7-th, 8-th, 12-th, 14-th, 21-st and 28-th as well as on December 5-th, 2018, several manual therapy sessions were carried out which involved adjustments to the cervical and thoracic spine with special attention being paid to the removal of spinal blockages at Th4/Th5.

The patient's condition improved significantly. Pain and constant discomfort in the left side of her chest ceased; the extrasystoles disappeared; weakness, trembling, feeling of choking, chills, attacks of groundless fear all went away. The patient's blood pressure stabilized at 120/80. The patient visited MC "Ukrainian Trauma Center" again on May 20-th, 2019 after having suffered a viral infection with complaints of renewed pain in the left side of her chest and extrasystoles. On May 20-th and 24-th, two manual therapy sessions were carried out to make necessary adjustments to the patient's thoracic spine. The patient's condition stabilized, pain and extrasystoles disappeared and panic attacks never occurred again after the first stage of treatment performed back in 2018.

\section{Clinical case No. 4}

Male patient V, 44 y.o., manager. First presented to MC "Ukrainian Trauma Center" on May 28-th, 2018. Diagnosis: coronary heart disease, atherosclerotic cardiosclerosis, stage 1 arterial hypertension, vertebrogenic cervical thoracic pain disorder, stage 1 discirculatory encephalopathy with autonomic dysfunction accompanied by anxiety/phobic manifestations and panic attacks.

Complaints: chest pain in the heart region, attacks of fear, weakness, back pain along the spine, sensation of shortness of breath, trembling (presence of 5 diagnostic criteria for panic attacks specified in DSM-5 and ICD-11).

Medical history: became ill on May 10-th, 2018, the aforementioned complaints arose suddenly after staying in a draught for a short while. During the period from May 20-th until May 27-th, the patient was admitted to the Kyiv Regional Cardiology Clinic where he received his diagnosis followed by medication and physiotherapy treatment. The patient was discharged from the hospital in satisfactory condition and presented to MC "Ukrainian Trauma Center" on May 28-th, 2018 seeking further treatment.

Clinical data: a spinal MRI demonstrated degenerative and dystrophic changes complicated by: intervertebral disc protrusions at $\mathrm{C} 3-\mathrm{C} 4$ in the cervical region of the spine; posterior protrusions at Th5-Th9 \& Th11-Th12 levels of the thoracic spine compressing the dural sac; vertebrogenic lumbalgia in L2-L3, L3-L4, L4-L5 and L5-S1 segments, intervertebral disc protrusions at L2-L5 and an intervertebral disc herniation at the level of L5-S1 all in the lumbosacral spine.

Treatment: on May 28-th and 30-th, as well as on June 1-st, 6-th, 8-th, 15-th, 22-nd and 29-th, followed by July 6-th and 13-th, the patient was subjected to a series of manual therapy sessions which involved removing spinal blockages at Th5 \& L4 levels through mobilization and manipulation techniques.

The patient's condition improved significantly: chest pain in the heart region, back pain, trembling, shortness of breath, weakness, attacks of fear all went away; the blood pressure stabilized at 120/80. On June 3-rd, 2019, the patient presented to MC "Ukrainian Trauma Center" for repeated treatment complaining of pain in the lumbosacral spine after lifting a heavy object. He was subjected to a session of manual therapy which involved necessary adjustments to his spine and successfully relieved the pain. In all this time, the patient hasn't experienced any repeated panic attacks.

\section{Clinical case No. 5}

Male patient M, 36 y.o., insurance company employee. First presented to MC "Ukrainian Trauma Center" in April 2016. Diagnosis: spinal osteochondrosis with a primary lesion of the thoracic and lumbosacral spine accompanied by radicular syndrome, vertebrogenic thoracalgia, stage 1 arterial hypertension, cardiac-type vegetative-vascular dystonia syndrome (vascular dysautonomia), panic attacks, chronic cholecystic pancreatitis.

Complaints: pain and constant discomfort in the chest, weakness, increased anxiety, attacks of fear, nausea and abdominal distress, vasospasms with abrupt elevations of blood pressure up to 
200/110, paroxysmal palpitations, trembling, fear of imminent death (presence of 7 diagnostic criteria for panic attacks specified in DSM-5 and ICD-11).

Medical history: became ill as a child; has been experiencing increased anxiety and fear for as long as he can remember; chest pain, sudden nausea and abdominal distress first appeared at the age of 13 y.o.; panic attacks started at the age of 17 y.o. and are usually accompanied by tachycardia and elevated blood pressure. On multiple occasions, this individual was admitted to various specialized medical institutions where he received medication and psychotherapy treatment an in-patient. After short periods of remission, panic attacks resume and occur with an interval of 5-10 days. The patient first presented to MC "Ukrainian Trauma Center" seeking treatment for his vertebrogenic thoracalgia condition.

Clinical data: a spinal MRI revealed degenerative and dystrophic changes in the thoracic spine complicated by dorsal protrusions of the intervertebral disks Th3-Th4, Th9-Th10, Th10-Th11 \& Th11Th12. 24-hour Holter monitoring of the heart repeatedly recorded horizontal/downward sloping ST depressions with a duration of 5-10 minutes each, accompanied by an increased heart rate of 110-159 beats per minute and compressive pain behind the sternum. Contrast coronarography revealed no significant ischemia of vascular vessels.

Treatment: on April 11-th, 13-th, 15-th, 22-nd and 29-th, 2016 a series of manual therapy sessions was carried out which involved removing spinal blockages at C4, C5 and occasionally C6 levels through mobilization and manipulation techniques.

The patient's condition improved. Chest pain, weakness, increased anxiety, palpitation, attacks of fear, nausea and abdominal distress all went away; the blood pressure stabilized at 120/80. The patient repeatedly presented to MC "Ukrainian Trauma Center" with renewed symptoms, including panic attacks, in 2 months. Remission occurred after two sessions of manual therapy but in another 2 months the symptoms returned again. Since 2016, the patient has been regularly receiving treatment at MC "Ukrainian Trauma Center" every 1-2 month, the period for which remission can be achieved with one or two sessions of manual therapy. The patient has abandoned medication treatment as ineffective for his condition.

Clinical case No. 6

Mail patient A, 58 y.o., swimming coach, master of sports. First presented to MC "Ukrainian Trauma Center" in June 2017. Diagnosis: vertebrogenic spinal disorder with a primary lesion of the thoracic spine (thoracalgia), panic attacks, aortic and mitral valve insufficiency, left ventricular hypertrophy.

Complaints: chest pain (in the upper chest and behind the sternum in the scapula area) and constant discomfort, weakness, extrasystoles, shortness of breath and smothering, increased anxiety, fear of dying while asleep, vasospasms with abrupt elevations of blood pressure up to 180/110, paroxysmal palpitations, feeling of imminent death (presence of 6 diagnostic criteria for panic attacks specified in DSM-5 and ICD-11).

Medical history: the patient has been suffering from his present condition since the age of 50 . He believes anxiety and fear to be caused by sudden attacks of extrasystoles and tachycardia as well as elevated blood pressure. On multiple occasions, the patient was medically examined and treated for cardiological diseases. First presented to MC "Ukrainian Trauma Center" seeking treatment for his vertebrogenic spinal disorder.

Clinical data: a spinal MRI revealed degenerative and dystrophic changes in the thoracic spine complicated by dorsal protrusions of the intervertebral disks Th3-Th4 \& Th10-Th11. Heart ultrasound and ECG study showed extrasystoles, aortic/mitral valve insufficiency and left ventricular hypertrophy.

Treatment: on June 4-th, 5-th, 9-th and 16-th, 2017 a series of manual therapy sessions was carried out which involved removing spinal blockages at C4 \& Th4 levels through mobilization and manipulation techniques.

After the above treatment, the patient's condition improved; chest pain, palpitations, weakness, extrasystoles, shortness of breath and smothering, anxiety, fear and vasospasms all disappeared, the blood pressure returned to normal settling at 120/80. On May 24-th, 2019, the patient made a repeated visit to MC "Ukrainian Trauma Center" with complaints of renewed chest pain, palpitations, extrasystoles and increased anxiety. A single session of manual therapy eliminated all his renewed symptoms. No panic attacks occurred in two years of observation after the initial treatment.

Clinical case No. 7

Female patient N., 68 y.o., working retiree. First presented to MC "Ukrainian Trauma Center" on January 11-th, 2016. Diagnosis: stage 3 hypertension with crises of a mixed sympatho-adrenal 
nature, cerebral atherosclerosis, suffered an ischemic stroke in 2010 and a closed head injury (hemorrhagic contusion of the basal regions of the right frontal brain lobe) in 2011, hypertensive heart (left ventricular concentric remodeling), underwent a cholecystectomy for cholelithiasis in 1994, nodular goiter with signs of autoimmune thyroiditis, chronic pyelonephritis, Crohn's disease, vertebrogenic spinal disorder (vertebrogenic cervicothoracolumbar pain disorder).

Complaints: pain and constant discomfort in the upper chest and behind the sternum, pain in the lumbosacral spine, palpitations, tremor, weakness, extrasystoles, shortness of breath and smothering, chills, blood pressure in the range of 220/120 - 280/140, dizziness and unsteadiness, nausea and abdominal distress, derealization and depersonalization, feeling of imminent inevitable dying (presence of 10 diagnostic criteria for panic attacks specified in DSM-5 and ICD-11).

Medical history: has been ill since 20 y.o., was first diagnosed with vegetative-vascular dystonia (vascular dysautonomia) at that same age. High blood pressure not controllable by medication has always been this patient's main complaint. Was hospitalized on multiple occasions and treated as an in-patient for her conditions. Medication treatment in this patient has always been of very limited and short-lasting effect, often even triggering inadequate response and causing adverse allergic reactions including anaphylactic shock. In 1994, the patient underwent a cholecystectomy for cholelithiasis. In 2010, with a blood pressure of 280/160, she suffered an ischemic stroke and in 2011 sustained a closed head injury (hemorrhagic contusion of the basal regions of the right frontal brain lobe) in a vehicle-pedestrian collision. The patient first presented to MC "Ukrainian Trauma Center" on January 11-th, 2016 seeking to continue her treatment after being discharged from a cardiology clinic without any relief.

Clinical data: a spinal MRI revealed degenerative and dystrophic changes complicated by: dorsal protrusions of the intervertebral disks $\mathrm{C} 2-\mathrm{C} 3, \mathrm{C} 4-\mathrm{C} 5, \mathrm{C} 5-\mathrm{C} 6$ and intervertebral disc herniations at the level of $\mathrm{C} 3-\mathrm{C} 4 \& \mathrm{C} 6-\mathrm{C} 7$ in the cervical spine; dorsal protrusions of the intervertebral disks Th4Th6, Th6-Th7, Th7-Th8 \& Th12-L1 in the thoracic spine; diffuse circular protrusions of the intervertebral disks L1-L2, L2-L3, L3-L4, a right-sided paramedian extrusion at L4-L5 and a rightsided paramedian protrusion at L5-S1 in the lumbosacral spine.

Treatment: considering the immensity and complexity of the diagnosis, as well as the subject of this paper, the focus will be on the treatment of the patient's panic attacks. The patient has been observed at MC "Ukrainian Trauma Center" for the last 3.5 years. In all these years, there have been only three episodes that can be qualified as panic attacks, including the patient's first visit to MC "UTC". On January 11-th, 13-th, 15-th, 18-th and 22-nd, 2016, a series of manual therapy sessions was carried out which involved removing spinal blockages at C3, C4 \& Th5 levels through mobilization and manipulation techniques.

The patient's condition improved, her pain and constant discomfort in the chest as well as palpitations and extrasystoles decreased, dizziness and unsteadiness, nausea and abdominal distress, derealization and depersonalization, feeling of imminent inevitable dying all disappeared. Another panic attack occurred on March 24-th, 2016 due to an incorrect kinesiology treatment procedure which immediately caused dizziness and unsteadiness, nausea and abdominal distress, shortness of breath and smothering, derealization and depersonalization and finally an abrupt elevation of blood pressure from 180/110 up to 280/130. Intravenous and infusion therapy failed to stop the panic attack. On March 25-th, 28-th and 30-th followed by April 1-st, 4-th and 6-th, a series of manual therapy sessions was carried out which involved removing spinal blockages at C3 \& C4 levels through mobilization and manipulation techniques. This approach helped stop the panic attack. The third panic attack occurred on June 13-th, 2019 and was caused by the patient's staying in a draught for a short while during summer heat. In two hours after changing her position from vertical to horizontal, the patient suddenly started experiencing severe dizziness, nausea, acute abdominal distress, severe palpitations, trembling, a feeling of derealization and depersonalization, an instantaneous elevation of blood pressure from 160/90 up to 260/130. This final panic attack lasted for three hours and it took two sessions of manual therapy on June 14-th and 17-th to completely eliminate it by removing C 3 \& C4 spinal blockages through mobilization and manipulation techniques.

Conclusions. Manual therapy combined with shock wave therapy allows helping patients with treatment-resistant panic attacks who have not received desired treatment results in specialized medical institutions that employ conventional medication and psychological therapy-based methods of treatment. This method is cost effective, does not require lengthy and expensive medical examinations and allows to quickly bring the patient back to normal life at a minimal financial and psychological cost. 


\section{REFERENCES}

1. American Psychiatric Association (2013), Diagnostic and Statistical Manual of Mental Disorders (5th ed.), Arlington: American Psychiatric Publishing, pp. 214-217, ISBN 978-0890425558.

2. "Anxiety Disorders". NIMH. March 2016. Archived from the original on 29 September 2016. Retrieved 1 October 2016.

3. Anxiety: management of anxiety (panic disorder, with or without agoraphobia, and generalised anxiety disorder) in adults in primary, secondary and community care. National Institute for Health and Clinical Excellence. Clinical Guideline 22. Issue date: April 2007 "Archived copy" (PDF). Archived from the original (PDF) on 24 August 2009. Retrieved 2009-07-21. ISBN 1-84629-400-2.

4. Bandelow, Borwin; Domschke, Katharina; Baldwin, David (2013). Panic Disorder and Agoraphobia. OUP Oxford. p. Chapter 1. ISBN 9780191004261. Archived from the original on 20 December 2016.

5. Bourne, E. (2005). The Anxiety and Phobia Workbook, 4th Edition: New Harbinger Press.

6. Breathing in and out of a paper bag Archived 21 October 2007 at the Wayback Machine.

7. Cramer, K., Post, T., \& Behr, M. (January 1989). "Cognitive Restructuring Ability, Teacher Guidance and Perceptual Distracter Tasks: An Aptitude Treatment Interaction Study". Archived from the original on 22 December 2010. Retrieved 2010-11-19.

8. Craske, Michelle (30 September 2011). "Psychotherapy for panic disorder"

9. Craske, MG; Stein, MB (24 June 2016). "Anxiety". Lancet. 388 (10063): 3048-3059. doi:10.1016/S01406736(16)30381-6. PMID 27349358.

10. Freire, Rafael C.; Zugliani, Morena M.; Garcia, Rafael F.; Nardi, Antonio E. (2016). "Treatment-resistant panic disorder: a systematic review". Expert Opinion on Pharmacotherapy. 17 (2): 159168. doi:10.1517/14656566.2016.1109628. ISSN 1744-7666. PMID 26635099.

11. Geddes, John; Price, Jonathan; McKnight, Rebecca (2012). Psychiatry. OUP Oxford. p. 298. ISBN 9780199233960. Archived from the original on 4 October 2016.

12. Ghadri, Jelena-Rima; et al. (June 7, 2018). "International Expert Consensus Document on Takotsubo Syndrome (Part I): Clinical Characteristics, Diagnostic Criteria, and Pathophysiology". European Heart Journal. 39 (22): 2032-2046. doi:10.1093/eurheartj/ehy076.

13. "Hyperventilation Syndrome]". 28 November 2016. Archived from the original on 13 July 2017. Retrieved 2017-09-18.

14. ICD-11 for Mortality and Morbidity Statistics (Version: 04/2019) https://icd.who.int/browse11/lm/en/\#/http://id.who.int/icd/entity/1707067746.

15. Lewit K., Sachse J., Janda V. (1993) Manual Medicine. - M.: Medicine.

16. Marchesi, Carlo (2008). "Pharmacological management of panic disorder". Neuropsychiatric Disease and Treatment. 4 (1): 93-106. PMC 2515914. PMID 18728820.

17. Meuret, AE; Ritz, T (October 2010). "Hyperventilation in panic disorder and asthma: empirical evidence and clinical strategies". International Journal of Psychophysiology: Official Journal of the International Organization of Psychophysiology. 78 (1): 68-79. doi:10.1016/j.ijpsycho.2010.05.006. PMC 2937087. PMI D 20685222.

18. Milrod, B.; Leon, A. C.; et al. (2007). "A randomized controlled clinical trial of psychoanalytic psychotherapy for panic disorder". American Journal of Psychiatry. 164 (2): 265272. doi:10.1176/ajp.2007.164.2.265. PMID 17267789.

19. "Panic Disorder: When Fear Overwhelms". NIMH. 2013. Archived from the original on 4 October 2016. Retrieved 1 October 2016.

20. Roth, WT (2010). "Diversity of effective treatments of panic attacks: what do they have in common?". Depression and Anxiety. 27 (1): 5-11. doi:10.1002/da.20601. PMID 20049938.

21. Sergii Frank. (2019) Experience in treating long-term effects of concussions and PTSD using manual therapy combined with shock wave therapy. World Science. 4(44), Vol.2. doi: 10.31435/rsglobal_ws/30042019/6470.

22. Taylor, Barr (2006). "Panic disorder". BMJ. 332 (7547): 951-955. doi:10.1136/bmj.332.7547.951 